\title{
Analysis and Design of Low Pass Filter using Unit Cell based on CRLH Transmission Line
}

\author{
Lei Yang \\ Department of Telecommunication Engineering \\ Jeju National University, Jeju, Korea \\ Doo-Yeong Yang \\ Department of Telecommunication Engineering \\ Jeju National University, Jeju, Korea
}

\begin{abstract}
In this paper, a unit cell for low pass filter design by using composite right and left-handed transmission line in conductor-backed coplanar waveguide is proposed. The characteristics of the unit cell are analyzed in order to design a low pass filter in small sizes. By changing the sizes of the unit cell, the parameters of right-handed and left-handed immittance components are changed and the desired characteristics of the unit cell are achieved. The equivalent circuit of the unit cell is extracted and analyzed either. As a result, the simulation results of the unit cell and the equivalent circuit are almost identified. The movement and energy distributions of electromagnetic field are shown to confirm the property of the unit cell. In the end, a low pass filter is demonstrated by cascading three proposed unit cells, which shows cutoff frequency of $1.53 \mathrm{GHz}$ and deep attenuation from $2.23 \mathrm{GHz}$ to $4.49 \mathrm{GHz}$ lower than $50 d B$.
\end{abstract}

Keywords: Composite Right and Left-Handed Transmission Line, Conductor Backed Coplanar Waveguide, Unit Cell, Low Pass Filter.

\section{INTRODUCTION}

Recently, left-handed materials (LHMs) have concentrated extensive attention in many areas. This special artificial material with simultaneously negative values of $\varepsilon$ and $\mu$ was presented in 1967 by the Russian physicist Viktor Veselago [1], and demonstrated experimentally until 2000 [2]. Since LHMs are actually homogeneous structures, artificial LH transmission line can be constructed effectively. The advantages of LH transmission line are high frequency selectivity, low loss and wide bandwidth, as well as it can be implemented in planar configurations, compatible with modern microwave integrated circuits (MICs) [3]. So they can be widely used for the efficient design of microwave applications, in order to get the target of miniaturization, low cost and low power loss. But a purely LH (PLH) structure does not exist in fact because parasitic elements exist, and then that was the motivation for the introduction of the term "composite right and left-handed (CRLH)" [4]-[6]. A CRLH model [4] has both a left-handed (LH: $\mathrm{L}_{\mathrm{L}}, \mathrm{C}_{\mathrm{L}}$ ) range, which is due to the effect of the loading elements, and a right-handed ( $\left.R H: L_{R}, C_{R}\right)$ range, which is due

\footnotetext{
* Corresponding author,Email: christinayang2@hotmail.com Manuscript received Mar. 13, 2012; revised Jul 06, 2012; accepted Jul 16, 2012
}

to parasitic elements of the transmission line. On CRLH structure, low pass filter (LPF), high pass filter (HPF), band pass filter (BPF) and band stop filter (BSF) can be designed by tuning to the RH and LH contributions [7], [8].

Filters are essential components in precision measuring instruments, which are commonly used in testing RF communication products. The development of precision measuring instruments requires filters to have a compact size and low insertion loss. Among the ways of configuring filters, which are used in high precision measuring instruments without high power, quarter wavelength microstrip line is commonly used. However, filters designed by meta unit cell structure, which is basically eighth wavelength, can be smaller in size and the insertion loss can be decreased. In the respect of designing filters in microwave integrated circuits (MICs) or monolithic microwave integrated circuits (MMICs), coplanar waveguide (CPW) is more suitable because of its advantages, such as the simplicity of fabrication, easy mounting of active and passive devices in series and shunt, easy connections without via hole and possible size reduction [9], [10]. Conductor-backed CPW (CBCPW) is similar to conventional $\mathrm{CPW}$, which is with an additional lower ground plane. The lower ground plane of $\mathrm{CBCPW}$ increases mechanical strength of the circuit and gets cooling effect for circuits with active 
devices. Moreover, CBCPW gets less dispersive than CPW [9], [11], [12].

In this paper, in order to obtain a low pass filter with cutoff frequency at $1.5 \mathrm{GHz}$ and insertion loss of $1.2 \mathrm{~dB}$, a unit cell with low-pass property is proposed by using CRLH metamaterials (MTMs) in CBCPW transmission line. And the equivalent circuit of the unit cell is extracted to analyze the performances of the unit cell. Also, the movement and energy distribution of electromagnetic field are shown to prove the property of CRLH MTMs. Then three proposed unit cells are connected in series to demonstrate a low pass filter, which can obtain a cutoff frequency at the $1.53 \mathrm{GHz}$ and deep attenuation from $2.23 \mathrm{GHz}$ to $4.49 \mathrm{GHz}$ lower than $-50 \mathrm{~dB}$.

\section{UNIT CELL USING CRLH CBCPW STRUCTURE}

The geometric configuration of the proposed unit cell of CRLH CBCPW transmission line is shown in Fig.1. The configuration consists of a dielectric substrate between two metallic layers; the top layer is composed by two ground planes and signal transmission line, and the bottom layer is a ground plane only. This structure is designed on printed circuit board with relative dielectric constant of 2.5 and thickness of $0.787 \mathrm{~mm}$, in order to analyze the performances of the proposed unit cell structure quantitatively. The width of feeder line $w$ is $8.74 \mathrm{~mm}$, and the gap width between feeder line and the ground plane $\mathrm{G}$ is $0.3 \mathrm{~mm}$, which are calculated by the tool Linecalc in advanced design system (ADS), responding to $50 \Omega$ characteristics impedance of CBCPW structure.

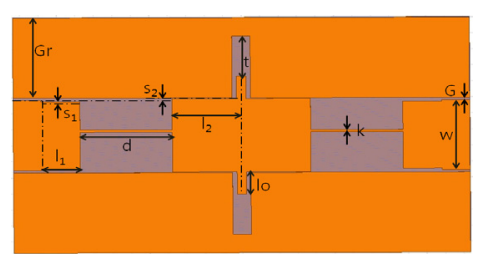

Fig. 1. Physical configuration of the unit cell CRLH CBCPW. $\left(\mathrm{Gr}=10 \mathrm{~mm}, 1_{1}=4.23 \mathrm{~mm}, 1_{2}=7.49 \mathrm{~mm}, \mathrm{~d}=10 \mathrm{~mm}, \mathrm{~s}_{1}=0.2 \mathrm{~mm}\right.$, $\left.\mathrm{s}_{2}=0.2 \mathrm{~mm}, \mathrm{t}=5 \mathrm{~mm}, \mathrm{lo}=3 \mathrm{~mm}, \mathrm{k}=0.25 \mathrm{~mm}\right)$

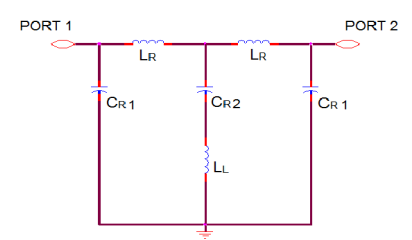

(a)

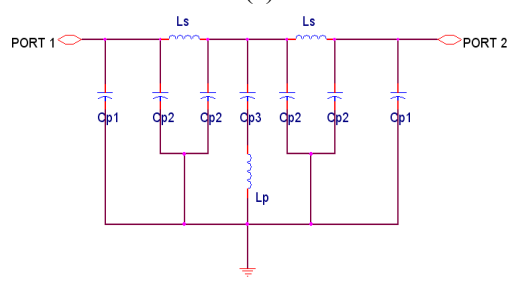

(b)

Fig. 2. Equivalent circuit of the unit cell (a) Approximate equivalent circuit. (b) Detailed equivalent circuit.
As shown in Fig.1, the input port is symmetric with the output port, based on the central axis of the transmission line. When the signal transmits through the broad transmission line, electrical energy is preserved and the parallel component $C_{R}$ is generated. Then the signal transmits through the narrow transmission line, magnetic energy is preserved and the series component $L_{R}$ is created. According as the signal transmits along the transmission line of the same geometry repeatedly, and those components are generated again. Between the broad transmission lines, coupling capacitance $\mathrm{C}_{\mathrm{L}}$ can be generated when the parameter $d$ is small enough. And $\mathrm{L}_{\mathrm{L}}$ can be achieved along the transmission lines which tend to the ground planes. By tuning the sizes of $\mathrm{w}, \mathrm{l}_{1}, \mathrm{l}_{2}, \mathrm{~d}, \mathrm{k}, \mathrm{s}_{1}, \mathrm{~s}_{2}$, lo and the cutoff frequency and the characteristics of pass-band and stop-band can be changed. In other words, the values of components $L_{R}$, $C_{R}, L_{L}, C_{L}$, which are shown in Fig.2 (a), change with the sizes of the unit cell. As a result, the $\mathrm{RH}\left(\mathrm{L}_{\mathrm{R}}, \mathrm{C}_{\mathrm{R}}\right)$ characteristic dominates on the unit cell of LPF, and the LH $\left(\mathrm{L}_{\mathrm{L}}, \mathrm{C}_{\mathrm{L}}\right)$ characteristic is suppressed. As shown in Fig.2 (b), the equivalent circuit values of inductance $\left(\mathrm{L}_{\mathrm{R}}=\mathrm{L}_{\mathrm{s}}, \mathrm{L}_{\mathrm{L}}=\mathrm{L}_{\mathrm{p}}\right)$ and capacitance $\left(\mathrm{C}_{\mathrm{R} 1}=\mathrm{C}_{\mathrm{p} 1}+\mathrm{C}_{\mathrm{p} 2}, \mathrm{C}_{\mathrm{R} 2}=\mathrm{C}_{\mathrm{p} 2}+\mathrm{C}_{\mathrm{p} 2}+\mathrm{C}_{\mathrm{p} 3}\right)$ can be calculated according to [13] as

$$
\begin{aligned}
& L_{s}=\left.\frac{Z_{C} \sin \left(\frac{2 \pi}{\lambda_{g}} d\right)}{\omega_{0}} \approx \frac{Z_{C} 2 \pi d}{\lambda_{g} \omega_{0}}\right|_{d<\frac{\lambda_{g}}{8}}=\frac{Z_{C} d \sqrt{\varepsilon_{e f f}}}{c} \\
& L_{p}=\left.\frac{Z_{C} \sin \left(\frac{2 \pi}{\lambda_{g}} l o\right)}{2 \omega_{0}} \approx \frac{Z_{C} 2 \pi l o}{2 \lambda_{g} \omega_{0}}\right|_{l o<\frac{\lambda_{g}}{8}}=\frac{Z_{C} l o \sqrt{\varepsilon_{e f f}}}{2 c} \\
& C_{p 2}=\left.\frac{\tan \left(\frac{\pi}{\lambda_{g}} d\right)}{Z_{C} \omega_{0}} \approx \frac{\pi d}{Z_{C} \lambda_{g} \omega_{0}}\right|_{d<\frac{\lambda_{g}}{8}}=\frac{d \sqrt{\varepsilon_{e f f}}}{2 c Z_{C}} \\
& C_{p 1}=\varepsilon_{0}\left(\varepsilon_{e f f}+1\right) \frac{l_{1} \times\left(w-s_{1}\right)}{h} \\
& C_{p 3}=\varepsilon_{0}\left(\varepsilon_{e f f}+1\right) \frac{2 l_{2} \times\left(w+s_{2}\right)}{h}
\end{aligned}
$$

where $L_{s}$ and $L_{p}$ are created at the interval $d$ and the interval lo of narrow transmission lines. $\mathrm{L}_{\mathrm{p}}$ controls the skirt characteristics of low pass filter in the stop-band. And also, $\mathrm{C}_{\mathrm{p} 1}$ and $\mathrm{C}_{\mathrm{p} 3}$ are created between the interval $1_{1}$, the interval $2 l_{2}$ of the broad transmission lines and the bottom ground plane, respectively. The $\mathrm{C}_{\mathrm{p} 2}$ is created at the discontinuities of transmission lines with different width [13], but it has little effect on characteristics since the value is small. The $\mathrm{h}$ and $\mathrm{w}$ are the thickness of the dielectric substrate and the width of feeder line. The $\mathrm{c}$ is the velocity of light, $\varepsilon_{0}$ is the permittivity of free space and $\lambda_{\mathrm{g}}$ is the guided wavelength. The values of effective permittivity $\varepsilon_{\text {eff }}$ and characteristic impedance $Z_{C}$ can be calculated responding to the sizes of transmission line width and the gap width between transmission line and the ground plane in CBCPW structure by the tool Linecalc in ADS. For example, when the width of feeder line $\mathrm{w}$ is $8.74 \mathrm{~mm}$, the gap 
width between feeder line and the ground $G$ is $0.3 \mathrm{~mm}$, the result of effective permittivity $\varepsilon_{\text {eff }}$ is 2.182 . The calculated values of the equivalent elements result in $\mathrm{L}_{\mathrm{s}}=7.039 \mathrm{nH}, \mathrm{L}_{\mathrm{p}}=$ $0.3365 \mathrm{nH}, \mathrm{C}_{\mathrm{p} 1}=1.270 \mathrm{pF}, \mathrm{C}_{\mathrm{p} 2}=0.1544 \mathrm{pF}$, and $\mathrm{C}_{\mathrm{p} 3}=4.801 \mathrm{pF}$ at $\mathrm{f}_{0}=1.47 \mathrm{GHz}$.

\section{SIMULATION RESULTS OF UNIT CELL}

Simulations of the unit cell and the equivalent circuit are carried out by using HFSS and ADS separately. The simulation results are plotted in Fig.3. As shown in Fig.3, the unit cell of CRLH CBCPW transmission line exhibits a low-pass characteristic. By changing sizes of the unit cell, cut-off frequency and the characteristics of pass-band and stop-band can be changed to get the desired low-pass characteristics. Comparing the simulation results of the unit cell with the ones of the equivalent circuit, graphs of S-parameters get to similar forms and similar characteristics. As shown in Fig.3 (a), the cutoff frequency of the unit cell is $1.37 \mathrm{GHz}$ and of the equivalent circuit is $1.33 \mathrm{GHz}$. Therefore, the error rate of cutoff frequency is $3.13 \%$. Similarly, the error rate of cutoff frequency shows $4.48 \%, 5.37 \%$ and $7.17 \%$ in Fig. 3 (b), (c) and (d). The reason is that an error rate of the simulation result becomes bigger when the length of design variable $l_{1}$ approaches to $\lambda_{\mathrm{g}} / 8$.

Comparing these four figures, the S-parameter curves show different characteristics. The magnitude of $S_{21}$ curves in (a) and (b) is maximum flat in pass-band, but gets ripples in stop-band. Therefore, it can be seen as quasi-elliptic filter [14]. Moreover, the curves of (c) and (d) get ripples in both pass-band and stopband, which is similar with the characteristics of elliptic filter [14]. Overall, (b) gets the best characteristics that in pass-band the ripples of $\mathrm{S}_{11}$ are below $-17.78 \mathrm{~dB}$ and the maximum magnitude of $\mathrm{S}_{21}$ curve is flat and decreases monotonically. That is, the characteristics of the unit cell turn better when the lengths of $l_{1}, l_{2}$ approach to $\lambda_{\mathrm{g}} / 32$. Once the lengths of low pass filter stubs are being $\lambda_{\mathrm{g}} / 8$ [15] or $\lambda_{\mathrm{g}} / 4$ [16] in general, this unit cell is more advantageous to achieve miniaturization in filter design.

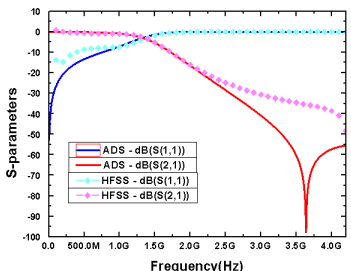

(a)

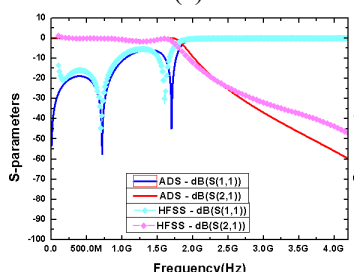

(c)

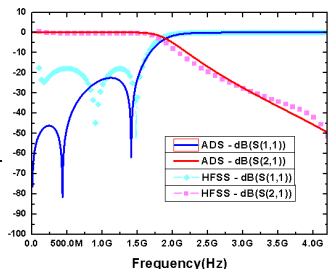

(b)

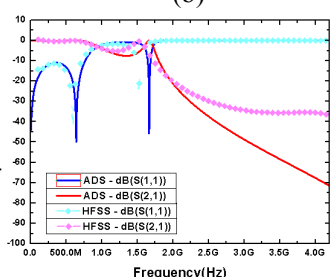

(d)
Fig. 3. Simulated S-parameters of the unit cell

(a) $1_{1}=\lambda_{\mathrm{g}} / 32,1_{2}=\lambda_{\mathrm{g}} / 16$. (b) $1_{1}=\lambda_{\mathrm{g}} / 32,1_{2}=\lambda_{\mathrm{g}} / 32$.

(c) $1_{1}=\lambda_{\mathrm{g}} / 16,1_{2}=\lambda_{\mathrm{g}} / 32$.(d) $1_{1}=\lambda_{\mathrm{g}} / 8,1_{2}=\lambda_{\mathrm{g}} / 32$.

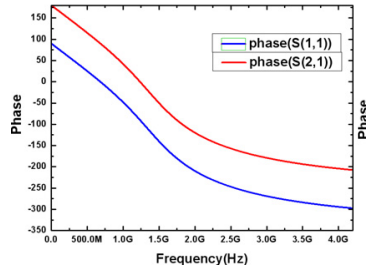

(a)

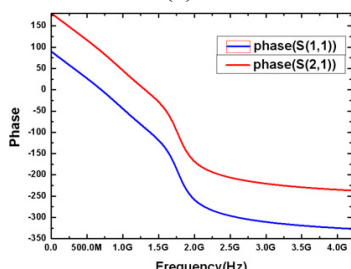

(c)

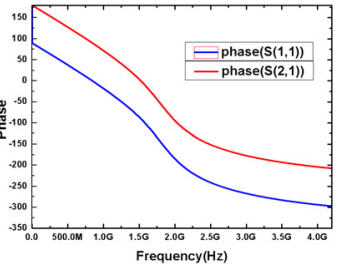

(b)

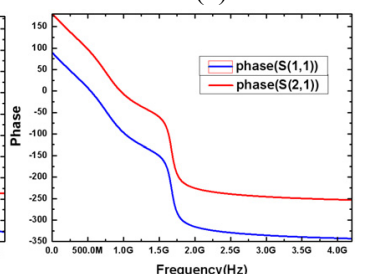

(d)
Fig. 4. Phase characteristics of the unit cell (a) $1_{1}=\lambda_{\mathrm{g}} / 32,1_{2}=\lambda_{\mathrm{g}} / 16$ (b) $1_{1}=\lambda_{\mathrm{g}} / 32,1_{2}=\lambda_{\mathrm{g}} / 32$. (c) $1_{1}=\lambda_{\mathrm{g}} / 16,1_{2}=\lambda_{\mathrm{g}} / 32$.(d) $1_{1}=\lambda_{\mathrm{g}} / 8,1_{2}=\lambda_{\mathrm{g}} / 32$.

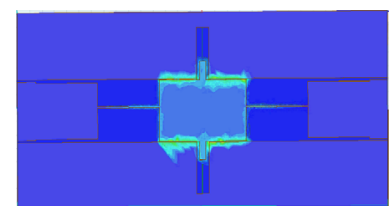

(a)

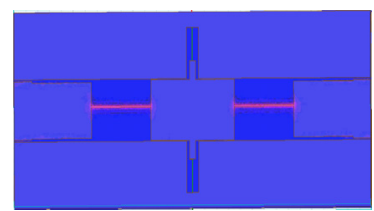

(b)
Fig. 5. Signal transmission of RH transmission line

(a) Energy distribution of electric field.

(b) Energy distribution of magnetic field.

As Fig.4, phase characteristics are shown to identify that if phase distortion occurs when signal transmits along the unit cell. As an ideal case, when the phase curve of $\mathrm{S}_{21}$ is a perfect straight line with a constant slope in pass-band, phase distortion of transmission signal does not occur at all. Compared to (c) and (d), the phase curves of (a) and (b) change linearly and get better characteristics. That is, the phase distortion in (c) and (d) occurs more than that in (a) and (b).

The energy distribution of electric field, corresponding to the accumulated energy distribution of the parallel circuit composition $C_{R}$, which is generated on the broad transmission line, is shown in Fig.5 (a). Because of the electromagnetic interaction, as time elapses, the electric energy turns to be magnetic energy, corresponding to the accumulated energy distribution of the series circuit component $L_{R}$, which is created on the narrow transmission line, is shown in Fig.5 (b). This process is repeated while the signal energy transmits from the input port to the output port. Therefore, the transformation and suppression of the input signal energy is achieved by the inductors and capacitors which build up the RH meta-unit cell. And according to the values of the inductors and capacitors, the unit cell shows the nature of low pass filter. Eventually, the RH meta-unit cell gets the characteristics of preserving the input electromagnetic energy and transmitting the energy to the output port without loss, as well as the characteristic of lowpass frequency selectivity. 


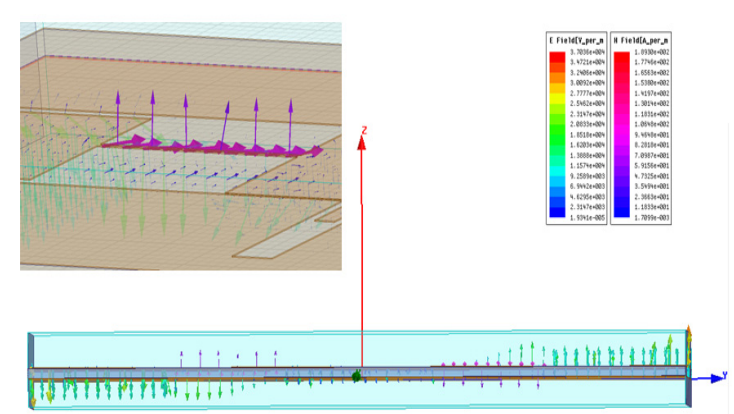

Fig. 6. Movement of electric field and magnetic field.

The movement of electric field and magnetic field can be seen in Fig.6. According to the direction of electric field of $-\mathrm{z}$ axis and magnetic field of $-\mathrm{x}$ axis, the direction of propagation waves can be determined to $+y$ axis.

\section{SIMULATION AND MEASUREMENT OF LOW PASS FILTER}

As analyzed above, the unit cell can be used for low pass filter design. In order to achieve the desired pass-band and the sufficient suppression in the stop-band, three unit cells are cascaded. Fig. 7 depicts the top layer physical configuration of the proposed low pass filter. The dimensions of the proposed filter are optimized by HFSS. The final optimized parameter values of the low pass filter are as follows: $1_{1}=9.01 \mathrm{~mm}, l_{2}=$ $7.72 \mathrm{~mm}, \mathrm{l}_{3}=9.03 \mathrm{~mm}, \mathrm{~d}=10.06 \mathrm{~mm}, \mathrm{~d}_{1}=10.02 \mathrm{~mm}, \mathrm{~s}_{1}=$ $0.11 \mathrm{~mm}, \mathrm{~s}_{2}=0.21 \mathrm{~mm}, \mathrm{~s}_{3}=0.01 \mathrm{~mm}, \mathrm{k}=0.24 \mathrm{~mm}, \mathrm{k}_{1}=1.5 \mathrm{~mm}$, $\mathrm{k}_{2}=0.35 \mathrm{~mm}, \mathrm{c}_{1}=0.53 \mathrm{~mm}, \mathrm{c}_{2}=0.06 \mathrm{~mm}, \mathrm{t}_{1}=0.5 \mathrm{~mm}, \mathrm{t}_{2}=$ $0.61 \mathrm{~mm}, \mathrm{lo}_{1}=2.12 \mathrm{~mm}, \mathrm{lo}_{2}=2.14 \mathrm{~mm}$.

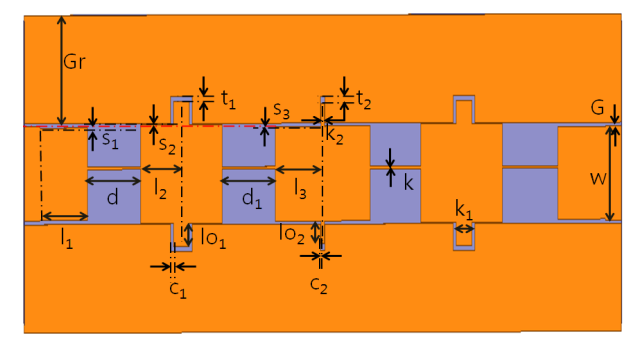

(a)

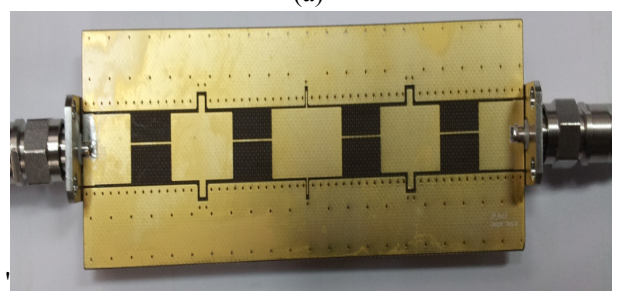

(b)

Fig. 7. Top layer physical configuration of the proposed low pass filter. (a) Simulated layout pattern. (b) Fabricated layout pattern.

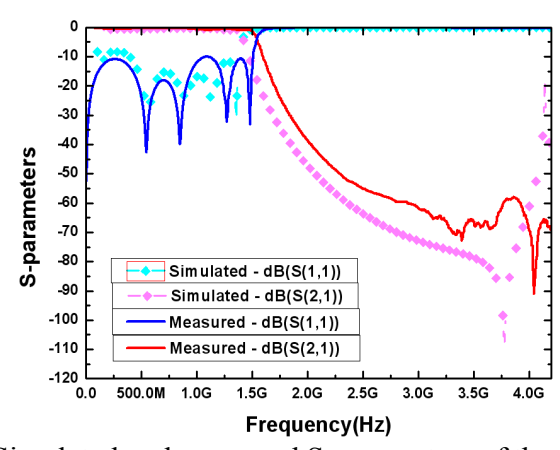

Fig. 8. Simulated and measured S-parameters of the proposed low pass filter.

The simulated and measured S-parameters of the proposed low pass filter are shown in Fig.8. The measured results show good agreement with the simulated ones, but the cutoff frequency does not match exactly. The experiment results demonstrate that the fabricated low pass filter has a $3 \mathrm{~dB}$ cutoff frequency at $1.53 \mathrm{GHz}$, right-shifted to $300 \mathrm{MHz}$. The ripples of S-parameter $S_{11}$ in pass-band are below $-10 \mathrm{~dB}$ and the $S_{21}$ is below $-50 \mathrm{~dB}$ from $2.23 \mathrm{GHz}$ to $4.49 \mathrm{GHz}$ in stop-band. However, spurious mode is appeared at $4.6 \mathrm{GHz}$ in stop-band because of the intrinsic property of CPW transmission line.

\section{CONCLUSIONS}

In summary, this paper focuses on investigating the unit cell of CRLH CBCPW transmission line, which shows lowpass property. The equivalent circuit and the simulated characteristics of CRLH CBCPW filter have been presented. As a result, the equivalent circuit shows similar characteristics with the unit cell. A low pass filter is demonstrated by cascading three unit cells. The cutoff frequency of the low pass filter is $1.53 \mathrm{GHz}$ with stop-band from $2.23 \mathrm{GHz}$ to $4.49 \mathrm{GHz}$ which is suppressed better than $-50 \mathrm{~dB}$.

\section{REFERENCES}

[1] V. Veselago, "The electrodynamics of substances with simultaneously negative values of $\varepsilon$ and $\mu$," Soviet Physics Uspekhi, vol. 10, no. 4, Feb. 1968, pp. 509-514.

[2] D. R. Smith, W. J. Padilla, D. C. Vier, S. C. NematNasser, and S. Schultz, "Composite medium with simultaneously negative permeability and permittivity," Phys. Rev. Lett., vol. 84, no. 18, May 2000, pp. 41844187.

[3] C. Caloz and T. Itoh, Electromagnetic metamaterials: transmission line theory and microwave applications, John Wiley \& Sons, Inc., 2006.

[4] C. Caloz and T. Itoh, "Novel microwave devices and structures based on the transmission line approach of meta-materials," Microwave Symposium Digest, IEEE MTT-S International, vol. 1, June 2003, pp. 195-198.

[5] A. Lai, T. Itoh and C. Caloz, "Composite right/lefthanded transmission line metamaterials," IEEE Microwave Magazine, Sept. 2004, pp. 34-50. 
[6] Qi Zhu and Shan-Jia Xu, "Composite right/left handed transmission line metamaterials and applications," Meta. 2008 International workshop, Nov. 2008, pp. 72-75.

[7] J. Garcia-Garcia, I. B. Vendik, B. Sans, D. Kholodnyak, P. Kapitanova, J. Bonache and F. Martin, "Miniaturization and optimization of planar microwave filters based on metamaterials," Micro. Conf. 2007, European, Oct. 2007, pp. 500-503.

[8] Pan Pan, Qun Wu, Fan-yi Meng, Fang Zhang and Jongchul Lee, "A novel compact left-handed transmission line with improved split-ring resonators," Micro. Conf. APMC 2007, Dec. 2007, pp. 1-4.

[9] Rainee N. Simons, Coplanar waveguide circuits, components, and systems, John Wiley \& Sons, Inc., 2001, pp. 1, 87-109.

[10] Cheng P. Wen, "Coplanar waveguide: a surface strip transmission line suitable for nonreciprocal gyromagnetic device applications," IEEE Trans. Micro. Theory Tech., vol. 17, no. 12, Dec 1969, pp. 1087-1090.

[11] Giovanni Ghione and Carlo U. Naldi, "Coplanar waveguides for MMIC applications: effect of upper shielding, conductor backing, finite-extent ground planes, and line-to-line coupling," IEEE Trans. Micro. Theory Tech., vol. 35, no. 3, Jan. 2003, pp. 260-267.

[12] Y.C. Shih and T. Itoh, "Analysis of conductor-backed coplanar waveguide," Electron. Lett., vol. 18, no. 12, June 1982, pp. 538-540.

[13] Jia-Sheng Hong, M. J. Lancaster, Microstrip filters for $R F /$ microwave applications, John Wiley \& Sons, Inc., 2001, pp. 97-99, 89-91.

[14] Dong-Ryong Kim, Seo-Kwoo Choi and Chang-Hoon Yoon, Modern network synthesis theory and filter design, Hongrueng Science Pub., 1998, pp. 77-82, 85-89.

[15] Kwangsik Park, Kwisoo Kim, Jongsik Lim and Dal Ahn, "A new open stubs structure of Kuroda low-pass filter using DGS", Wireless and Microwave Tech. Conf., WAMICON '09, April 2009, pp. 1-4, 20-21.

[16] Haojia Lin, Xiaoqun Chen, Xiaowei Shi, Lei Chen and Yanfu Bai, "A wide stopband CPW low pass filter using quarter wavelength stepped impedance resonators", ICMMT Conf.,, May 2010., pp. 62-65, 8-11.

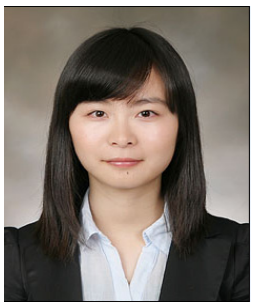

Lei Yang

She received the B.S. degree in telecommunication engineering from Jeju National University, Korea in 2011. From 2011, she is studying on master's course in telecommunication engineering, Jeju National University, Korea. Her main research interests include metamaterial, RF devices and wireless communication system.

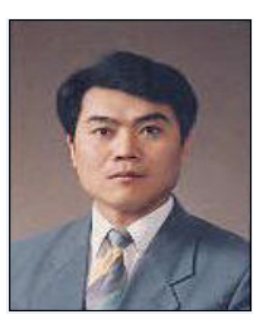

\section{Doo-Yeong Yang}

$\mathrm{He}$ received the B.S. degree in telecommunication engineering from Jeju National University, Korea in 1984, and M.S., Ph.D. degrees in electrical and telecommunication engineering from Hanyang University, Korea in 1989, 1992 respectively. Since 1992, he has been a professor in telecommunication engineering of Jeju National University in Korea. And also, from January 2001 to August 2002, he was an international research professor of Utah State University in USA.

His current research interests include RF devices, microwave circuits and wireless and satellite communication systems. 